\title{
Comparison of sublingual buprenorphine and intravenous morphine in reducing bone metastases associated pain in cancer patients
}

\author{
Seyed Mohammad Jamalian (1), Mohammad Sotodeh (2), Fathollah Mohaghegh (3)
}

(1) Department of Forensic Medicine and Poisoning, Arak University of Medical Sciences, Arak, Iran; (2). Department of Oncology, Arak University of Medical Sciences, Arak, Iran; (3) Department of Radiotherapy and Oncology, Arak University of Medical Sciences, Arak, Iran

This article is distributed under the terms of the Creative Commons Attribution Noncommercial License (CC BY-NC 4.0) which permits any noncommercial use, distribution, and reproduction in any medium, provided the original author(s) and source are credited.

\begin{abstract}
Bone metastases is one of the most common causes of pain in cancer patients and have a significant effect on their quality of life. The most common symptom of bone metastases is pain that gradually develops. Morphine is used to relieve pain in these patients, but poorly accepted due to its adverse events. Therefore, the current study was aimed to compare the effect of sublingual buprenorphine, with certainly lower complications with morphine. Fourth patients were divided into 2 groups. In group A, metastatic cancer patients received $2.5 \mathrm{mg}$ of intravenous morphine. Furthermore, in group B, sublingual tablet of buprenorphine (one-fourth of a $500 \mu \mathrm{g}$ tablet) was administered sublingually. Pain was measured 15, 30, and 45 minutes after the onset of pain using visual analog scale ruler. Based on the obtained data, two groups A and B were compared using SPSS 23 software. There was a significant difference between the patient's pain intensity after 15 and 30 minutes from the onset of pain in both groups. Due to the fact that the duration of the effect of morphine is 3-4 hours and the duration of the effect of sublingual buprenorphine is $6-8$ hours, morphine showed fast acting forms of opioids $(\mathrm{P}=0.001)$. The required dose level on the first day was similar in both groups and there was no statistically significant difference between the two groups. While on the second and third days, the median dose in group A (morphine) was greater than group B (buprenorphine), indicating prolonged duration of action for buprenorphine compared with morphine, thus requiring lower subsequent doses. The results of this study suggested that sublingual buprenorphine is a higher effective drug compared to intravenous morphine during and after operation. With regard to easy and painless administration, it seems that its use can be useful in controlling pain due to bone metastases in cancer patients.
\end{abstract}

Key Words: Sublingual Buprenorphine, morphine injection, pain, bone metastasis, cancer.

Bone metastases are one of the most common causes of pain in cancer patients and have a significant effect on their quality of life. ${ }^{1}$ It is difficult to accurately determine the incidence of bone metastases, but it is estimated that more than 100,000 patients in the United States are diagnosed annually with metastatic bone disease. ${ }^{2}$ The incidence of bone metastases is largely dependent on the initial site of cancer, and breast, prostate and lung cancers are the source of metastases for most cancer patients. ${ }^{3}$ Other tumors tend to have bone involvement include thyroid, melanoma and kidney, along with hematologic malignancies including myeloma and lymphoma that can also cause bone pain and destruction. ${ }^{4,5}$ The average survival rate of patients with bone metastases is of several months. Patients with bone metastases from breast or prostate cancer may have a longer survival time. Patients with breast or prostate cancer who have only bone metastases, have a mean survival from 2 to 4 years, as reported. ${ }^{6,7}$ Apart from whether the survival time is only of few months to several years, these patients generally need active treatment, for pain, mobility, hypercalcemia, pathological fractures, neurological disorders, anxiety, depression, spinal cord or neural roots compression, all symptoms that affect patient quality of life. ${ }^{8}$ The most common symptom of bone metastases is a localized pain that progresses gradually. This pain may be more severe at night and a good pain control in these patients can lead to a significant improvement in their quality of life. ${ }^{9}$ In many patients, pain control can be achieved using the World Health Organization (WHO) 
Table 1. Inclusion and exclusion criteria

\section{Inclusion criteria}

1. The pain caused by metastatic cancers including breast, lung, prostate

2. Indication of narcotic prescription according to reference sources

3. Age 15 to 70 years

\section{Exclusion criteria}

1. Pain caused by metastatic cancers other than breast, lung and prostate

2. Drug addicts

3. Use of other drugs and painkillers

4. Use of pain reduction techniques such as nerve block

5. Use of other drugs affecting the severity of the medication

Pain Control Protocol. ${ }^{10}$ Stage I is the use of non-opioid analgesics, such as asthenophenone or NSAIDs. The use of weak opioids, such as codeine, is recommended in stage II, and stage III involves the use of strong opioids such as morphine. ${ }^{11}$ Morphine is currently used to relieve pain in these patients. Morphine is poorly accepted by patients because of the addictive nature of the drug; however, patients are forced to use this drug for pain, which sometimes may be addicted to drug of the primary illness. Morphine, due to its intravenous administration, can cause atherosclerosis, obstruction, and vascular damage in these patients, and may also impair chemotherapy. ${ }^{12}$ Patients sometimes experience nausea, vomiting, and loss of consciousness and respiratory disorders after receiving morphine. ${ }^{13}$ The availability of an appropriate alternative medicine is one of the needs of these patients, able to demonstrate the analgesic effect of morphine, without effects and complications. Buprenorphine is a partial opioid agonist of the $\mu$ receptor, which exhibit no adverse effects due to antagonistic properties; it is currently used in the treatment of opiate dependence, and it has a special place in the treatment of these patients. ${ }^{14}$ Buprenorphine is used sublingually and can be well tolerated in cancer patients with digestive problem, leading to reduction of the need for the use of injectable analgesics in these patients. Now, the use of this drug, which is used only in the treatment of opioid dependence, can be potentially considered as a major step in the treatment of cancer pain, without concerning for dependence or complications. ${ }^{15}$

\section{Materials and Methods}

This study was a before and after clinical trial. All subjects were recruited using convenience sampling. The population of the study consisted of 40 metastatic cancer patients admitted to Ayatollah Khansari Hospital in Arak, Iran, who suffered from pain. Inclusion and exclusion creteria are listed in Table 1. Fourth patients were divided into 2 groups. In group A, metastatic cancer patients received $2.5 \mathrm{mg}$ of intravenous morphine, 5 minutes after the onset of pain, and then the pain was measured 15, 30, and 45 minutes after the onset of pain with visual analog scale (VAS) Ruler. Group B cancer patients received buprenorphine tablet sublingually (one fourth of the tablet of $500 \mu \mathrm{g}$ ), and pain was measured 15,30 , and 45 minutes after the onset of pain. In addition, patients in each of the two groups were asked to measure their pain using VAS Ruler every time they had pain. This procedure lasted for up to 3 days, and pain was relieved every time patients had pain. In case of complications, the type of complication was recorded. At the end of the pain phase, the patients of each group discontinued the narcotic drugs to an acceptable level for 3 days and in case of pain, pain was recorded. Based on the goals and data, the two groups A and B were compared and analyzed statistically.

\section{Sample size}

The sample size was estimated to be 20 for each group using the following formula:

$$
n=\frac{2 S_{p}{ }^{2}\left(Z_{1-\propto / 2}+Z_{1-\beta}\right)^{2}}{d^{2}}
$$

In the formula, the first type error $(\alpha)$ and second type error $(\beta)$ were considered as $5 \%$ and $2 \%$, respectively. Also, the standard deviation (SD) and the effect size (d), based on the values obtained by Abdolhosseinpour et al. ${ }^{16}$ were calculated as 0.5 and $13 \%$, respectively.

\section{Data analysis}

Data were analyzed using SPSS version 23 software. Furthermore, paired sample t-test, and chi-square were applied for statistical analysis.

\section{Ethical considerations}

The ethics committee of Arak University of Medical Sciences (code-2-173-93) approved this study. Prescribing of narcotic drugs in patients referred to pain is generally mentioned. Reference sources do not mention any differences between non-addicted patients and substance dependent patients. Scientific sources have recommended the use of narcotic buprenorphine or morphine, so this study is ethically consistent with the Helsinki declaration. According to the protocol, in the absence of control of pain with buprenorphine, the next step is to use morphine in a controlled manner.

\section{Results}

This study is a single-blind trial designed to compare the effect of sublingual buprenorphine and injectable morphine on the reduction of bone metastases pain in cancer patients. Forty patients were enrolled in this study. In the first stage, 20 patients were included in group A (morphine) and 20 patients in group B (buprenorphine). In the second stage, group A (buprenorphine) and group $\mathrm{B}$ (morphine) received the desired medication. In the morphine group, 3 were female and the rest were male; in the buprenorphine group, 4 were female and the rest 
Sublingual buprenorphine vs intravenous morphine in pain

Eur J Transl Myol 29 (2): 124-129, 2019

Table 2. Frequency distribution of drug use

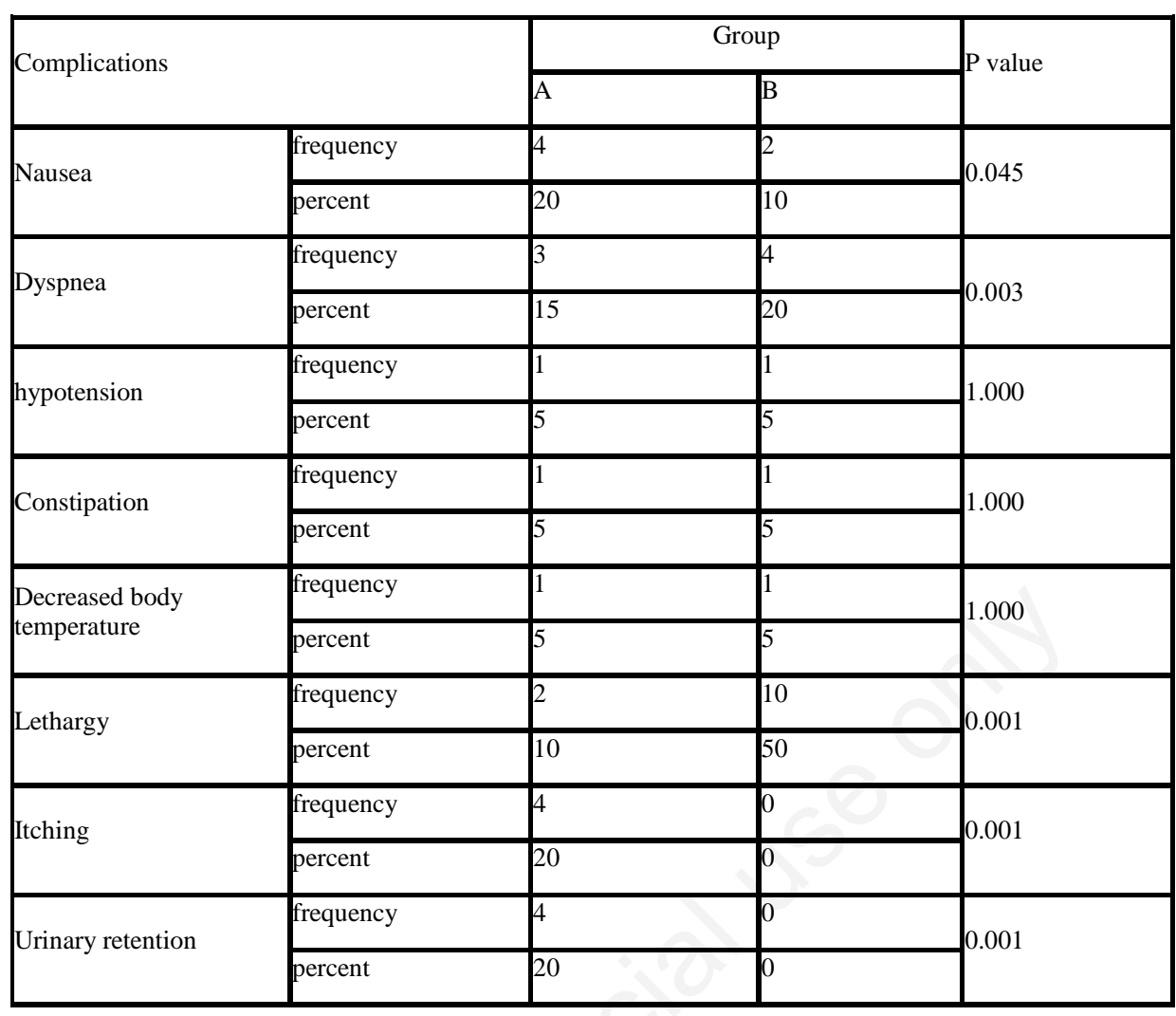

were male. The mean age in the group of morphine and buprenorphine was $52.06 \pm 7.95$ and $52.68 \pm 8.45$, respectively. Independent $\mathrm{T}$-test showed that the mean age of the subjects in the two groups was not statistically significant. Considering the results of Chi-square test, there was no significant difference between the two groups in terms of gender $(\mathrm{P}<0.05)$. As indicated in Table 2, the most frequent side effects of the drug in group A (morphine) were nausea $(20 \%, 4)$, itching $(20 \%$, $4)$, and urinary retention $(20 \%, 4)$, while, lethargy $(50 \%$, $10)$ and dyspnea (20\%, 4 persons) were the most frequent in group B (buprenorphine). Drug side effects such as nausea, itching and urinary retention were significantly higher in the morphine group as compared to buprenorphine group ( $\mathrm{P}<0.05$ ), and complications like dyspnea and lethargy were higher in the buprenorphine group in comparing with the morphine group ( $\mathrm{P}$ $<0.0005)$. Complications such as decreased pressure, constipation and decreased body temperature did not show any significant difference in both groups (Table 2). Pain was measured in both groups based on VAS ruler, the highest of which was determined to be 10 . The mean pain intensity based on VAS 15 minutes after the onsets of pain in group A (morphine) and group B (buprenorphine) was determined as $2.75 \pm 0.96$ and 4.50 \pm 1.57 , respectively. The mean pain in group A was 1.83 \pm 0.8930 minutes after the onsets of pain and in the group B (buprenorphine) was $2.30 \pm 1.83$.

Table 3. The mean pain intensity in the two groups

\begin{tabular}{|l|l||l|l|l|l|}
\hline \hline & group & number & mean & $\begin{array}{l}\text { Standard } \\
\text { deviation }\end{array}$ & P value \\
\hline Pain intensity 15 min & A & 20 & 2.75 & 0.96 & 0.001 \\
\cline { 2 - 6 } & B & 20 & 4.50 & 1.57 & 0.002 \\
\hline Pain intensity 30min & A & 20 & 0.80 & 0.89 & 0.246 \\
\cline { 2 - 6 } & B & 20 & 2.30 & 0.83 & \\
\hline Pain intensity 45 min & A & 20 & 0.05 & 0.52 & \\
\cline { 2 - 6 } & B & 20 & 0.20 & & \multirow{2}{*}{ B } \\
\hline
\end{tabular}




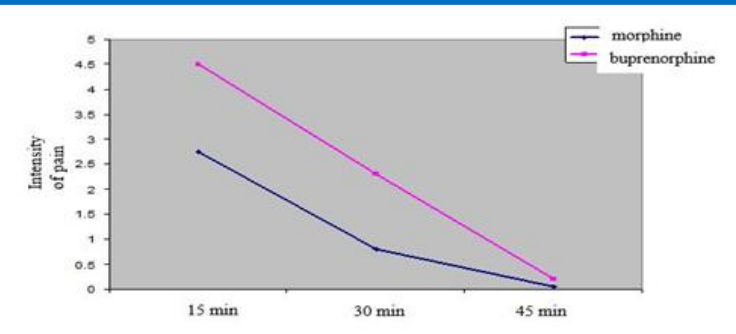

Fig 1. The mean pain intensity in the two groups

Independent $\mathrm{T}$ test revealed a significant difference between pain severity in 15 and 30 minutes after the onset of pain in both groups. The reason is that the duration of morphine action is approximately 3-4 hours and the duration of sublingual buprenorphine is 6-8 hours. Morphine therefore acts earlier in the first few minutes $(\mathrm{P}$ value $=0.001)$. Furthermore, the severity of pain in groups of $\mathrm{A}$ and $\mathrm{B}$ was determined as $0.05 \pm 0.022$ and $0.20 \pm 0.52$, respectively. However, no statistically significant difference between the two groups was found $(\mathrm{P}=0.246)$ (Table 3 and figure 1$)$. The mean of the required dose in group $\mathrm{A}$ (morphine) was calculated on the first, second and third day to be $1.50 \pm 0.76,2.10 \pm$ 0.71 and $2.05 \pm 0.75$, respectively. The mean of the required dose in Group B was also determined on the first, second and third day to be $1.55 \pm 0.60,1.20 \pm 0.83$, and $1.105 \pm 0.55$, respectively. Based on the results presented herein, independent T-test showed that the number of doses needed on the first day was the same in both groups and there was no significant difference between the two groups. While on the second and third days, the mean number of doses in group A (morphine) was greater than group B (buprenorphine), indicating that the duration of the effect of buprenorphine was greater than morphine and required a lower number of subsequent doses (Table 4 and Figure 2).

\section{Discussion}

When the cancer cells escape from the primary tumor, they move through lymph or blood ways to other parts of the body, where secondary tumors may develop. The

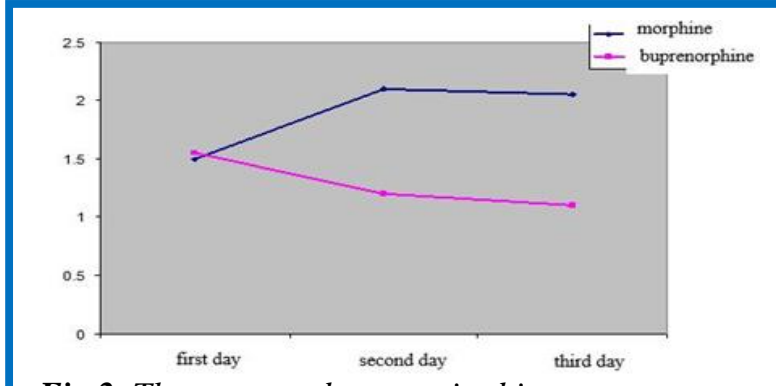

Fig 2. The average dose required in two groups

secondary tumor (metastasis) is of the same type of primary tumor. For example, if breast cancer spreads to bones, cancer cells in the bone are in fact breast cancer cells. ${ }^{17}$ When cancer cells, such as lung, breast, prostate, thyroid and/or others reach the bone, bone metastasis begin to develop. ${ }^{18}$ Clinical complications of the cancer include cardiovascular disease, respiratory system and urinary tract involvment, muscle weakness, osteoporosis, severe pain, etc. Despite all the progresses made in clinical pharmacology, proper control of pain in this case is still under discussion. ${ }^{19,20}$ Therefore, the aim of this study was to compare the effect of sublingual buprenorphine with injectable morphine on the reduction of pain associated with bone metastasis in cancer patients. The findings of this study showed the efficacy of sublingual buprenorphine in increasing the duration of analgesia and reducing the amount of pain more than intravenous morphine, as well as reduction of the drug side effects and its deprivation. Mean age in both groups was not statistically significant. There was no significant difference between the two groups regarding sex variable. The mean of pain intensity 15 and 30 minutes after the onset of pain in both groups did not show significant difference. The reason is that the duration of morphine activity is 3-4 hours and the duration of sublingual buprenorphine is 6-8 hours, thus morphine showed its effect in the early minutes indicating that the onset of the effect of the morphine drug is shorter than that of buprenorphine, but there was no significant difference between the two groups. The mean of the required dose on the first day was the same in both groups

Table 4. The mean dose required in the two groups

\begin{tabular}{|c|c|c|c|c||c|}
\hline & group & number & mean & $\begin{array}{c}\text { Standard } \\
\text { deviation }\end{array}$ & P value \\
\hline \multirow{2}{*}{ first day } & $\mathrm{A}$ & 20 & 1.5 & 0.76 & \multirow{2}{*}{0.819} \\
\cline { 2 - 6 } & $\mathrm{B}$ & 20 & 1.55 & 0.60 & \multirow{2}{*}{0.001} \\
\hline \multirow{2}{*}{ second day } & $\mathrm{A}$ & 20 & 2.10 & 0.71 & 0.83 \\
\cline { 2 - 6 } & $\mathrm{B}$ & 20 & 1.20 & 0.75 & 0.001 \\
\hline \multirow{2}{*}{ third day } & $\mathrm{A}$ & 20 & 2.05 & 0.55 \\
\cline { 2 - 6 } & $\mathrm{B}$ & 20 & 1.10 & \\
\hline
\end{tabular}


and there was no significant difference between the two groups. While on the second and third days, the mean number of doses in group A (morphine) was greater than group B (buprenorphine), indicating that the duration of the effect of buprenorphine was greater than morphine and required a lower number of subsequent doses. In two study conducted by Masson and Wallenstein et al., similar results were obtained when compared with our study, actually there were more remarkable effects of sublingual buprenorphine on pain control when compared with injected morphine. ${ }^{21,22}$ Abdolhosseinpour et al. has shown that sublingual buprenorphine can be used as an appropriate drug to reduce the intensity of postoperative pain. ${ }^{16}$ It is worth to emphasize that, in this study, buprenorphine is 33 times stronger than morphine. In addition, due to the high binding power of buprenorphine to nociceptive receptors, ${ }^{16,23}$ this drug can have longer analgesic effect. In our study, an increase in duration of analgesia and a reduction in the amount of pain with buprenorphine has been confirmed, probably indicating a different receptor affinity between morphine and its agonist-antagonist buprenorphine. On the other hand, the ease of administration of buprenorphine highlights the importance of its use in analgesia. In spite of this, Juhlin-Dannfelt et al. in 1995 observing higher rates of nausea associated with sublingual buprenorphine in comparison to pethidine, did not recommended this drug as an appropriate analgesic. ${ }^{24}$ Another study showed the significant effect of sublingual buprenorphine on the duration of analgesia in patients, which was consistent with duration of the analgesic effect of buprenorphine compared to morphine in our study. ${ }^{25}$ Abdolhosseinpour et al. have also assessed the effect of morphine injection versus sublingual buprenorphine on pain reduction after lumbar laminectomy and they concluded that sublingual buprenorphine could be used as an appropriate drug to reduce the intensity of postoperative pain after lumbar laminectomy, for its higher efficacy and easier use, when compared to morphine injection. In the present study, sublingual buprenorphine was more effective in reducing the severity of pain, avoiding complications of deprivation and drug intake than morphine, therefore, it is consistent with the outcome of aforementioned study. ${ }^{16}$ In another study by Capogna et al. ${ }^{26}$ involving 20 patients, the mean potency ratio between buprenorphine and morphine has be determined to be 32:1, and patients receiving buprenorphine had a much longer analgesia and more sedation score. In our study, the effect of morphine was greater than that of buprenorphine, but duration of sedation and efficacy of buprenorphine was higher when compared with morphine. ${ }^{26}$ In conclusion, our results indicate that buprenorphine is an effective sublingual agent compared to morphine injection and has a more prolonged analgesia. Because of its easier and painless administration, it seems that buprenorphine can be useful in controlling pain associated with bone metastases in cancer patients.

\section{List of acronyms}

visual analog scale - VAS

\section{Authors contributions}

All named authors played a substantial role in the conception and/or study design, data acquisition and/or analysis, as well as drafting of the manuscript, approval of the final version to be published, and agree the accountability for all aspects of accuracy and integrity of the work.

\section{Acknowledgments None.}

Funding No funding was obtained for this research project.

\section{Conflict of Interest}

The authors declare they have no financial, personal, or other conflicts of interest.

\section{Ethical Publication Statement}

We confirm that we have read the Journal's position on issues involved in ethical publication and affirm that this report is consistent with those guidelines.

\section{Corresponding Author}

Fathollah Mohaghegh, Department of Radiotherapy and Oncology, Arak University of Medical Sciences, Arak, Iran. Email: drfathollahmohaghegh@gmail.com and mohagheghfrt@yahoo.com

\section{E-mails of co-authors}

Seyed Mohammad Jamalian: jamalian_m@razi.tums.ac.ir Mohammad Sotodeh:mohammad_sotodeh@gmail.com

\section{References}

1. Von Moos R, Body JJ, Egerdie B, et al. Pain and analgesic use associated with skeletal-related events in patients with advanced cancer and bone metastases. Support Care Cancer 2016;24:1327-37.

2. Chin H, Kim J. Bone Metastasis: Concise Overview. Fed Pract 2015;32:24-30.

3. Macedo F, Ladeira K, Pinho F, et al. Bone Metastases: An Overview. Oncol Rev 2017;11:321.

4. Damsky W, Rosenbaum L, Bosenberg M. Decoding Melanoma Metastasis. Cancers (Basel) 2011;3: 126-63.

5. Forootan M, Tabatabaeefar M, Mosaffa N, et al. Investigating Esophageal Stent-Placement Outcomes in Patients with Inoperable Non-Cervical Esophageal Cancer. J Cancer 2018;9:213-8.

6. Svensson E, Christiansen CF, Ulrichsen SP, et al. Survival after bone metastasis by primary cancer type: a Danish population-based cohort study. BMJ Open 2017;7(9):e016022.

7. Liede A, Jerzak KJ, Hernandez RK, et al. The incidence of bone metastasis after early-stage breast cancer in Canada. Breast Cancer Res Treat 2016;156:587-95. 
8. Gulia A, Byregowda S, Kumar Panda P. Palliative Care in Musculoskeletal Oncology. Indian J Palliat Care 2016;22:244-51.

9. Lim FMY, Bobrowski A, Agarwal A, Silva MF. Use of corticosteroids for pain control in cancer patients with bone metastases: a comprehensive literature review. Curr Opin Support Palliat Care 2017;11:78-87.

10. Vargas-Schaffer G. Is the WHO analgesic ladder still valid? Twenty-four years of experience. Am Physician 2010;56:514-7.

11. Wiffen PJ, Wee B, Derry S, et al. Opioids for cancer pain - an overview of Cochrane reviews. Cochrane Database Syst Rev 2017;7:CD012592.

12. Schneider G, Voltz R, Gaertner J. Cancer Pain Management and Bone Metastases: An Update for the Clinician. Breast Care (Basel) 2012;7:113-20.

13. Young Whang B, Whan Jeong S, Gill Leem J, Ki Kim Y. Aspiration Pneumonitis Caused by Delayed Respiratory Depression Following Intrathecal Morphine Administration. Korean J Pain 2012;25:126-9.

14. Gowing L, Ali R, White JM, Mbewe D. Buprenorphine for managing opioid withdrawal. Cochrane Database Syst Rev 2017;2:CD002025.

15. Andrews CM, Abraham AJ, Grogan CM, et al. Impact of Medicaid Restrictions on Availability of Buprenorphine in Addiction Treatment Programs. Am J Public Health 2019;109:434-6.

16. Abdolhosseinpour H, Arbabi A, Moslem A, Tajik A. Efficacy of Morphine Injection Versus Sublingual Buprenorphine in Postoperative Pain After Lumbar Laminectomy. Horizon Med Sci 2010;16:20-4.

17. Scully OJ, Bay BH, Yip G, Yu Y. Breast cancer metastasis. Cancer Genomics Proteomics 2012;9:311-20.

18. Mavrogenis AF, Angelini A, Vottis C, et al. Modern Palliative Treatments for Metastatic Bone Disease: Awareness of Advantages, Disadvantages, and Guidance. Clin J Pain 2016;32:337-50.
19. Forootan M, Tabatabaeefar M, Maghsoodi N, et al. Adaptation of a Quality of Life Questionnaire for Iranian Patients with Esophageal Cancer. Indian J Palliat Care 2014;20:123-7.

20. Kamali A, Ashrafi TH, Rakei S, et al. A comparative study on the prophylactic effects of paracetamol and dexmedetomidine for controlling hemodynamics during surgery and postoperative pain in patients with laparoscopic cholecystectomy. Medicine (Baltimore) 2018;97:e13330.

21. Masson AH. Sublingual buprenorphine versus oral dihydrocodeine in post-operative pain. J Int Med Res 1981;9:506-10.

22. Wallenstein SL, Kaiko RF, Rogers AG, Houde RW. Clinical analgesic assay of sublingual buprenorphine and intramuscular morphine. NIDA Res Monogr 1982;41:288-93.

23. Alijanpour E, Rabiee M, Naziri F, Banihashem N, Rabiee O, Dorrudinia A. Comparison of sublingual buprenorphine and intravenous morphine on duration and severity of analgesia. Iranian Journal of Anesthesiology and Critical Care 2015;85(2).

24. Juhlin-Dannfelt M, Adamsen S, Olvon E, et al. Premedication with sublingual buprenorphine for out-patient arthroscopy: reduced need for postoperative pethidine but higher incidence of nausea. Acta Anaesthesiol Scand 1995;39:633-6.

25. Norouzi A, Talebi H, Alizadeh S, et al. PreAnesthetic Administration of Sublingual Buprenorphine forPostoperative Analgesia after Hemorrhoidectomy a Clinical Trial. Journal of Biological Sciences 2009;4:227-30.

26. Capogna G, Celleno D, Sebastiani M, et al. Continuous intravenous infusion with patientcontrolled anesthesia for postoperative analgesia in cesarean section: morphine versus buprenorphine. Minerva Anestesiol 1989;55:33-8.

Submission: February 02, 2019

Revision received: March 06, 2019

Acceptance: March 06, 2019 